\title{
Jornada "Enseñar matemáticas en aulas que incluyen alumnos con y sin discapacidad". 24 de Noviembre de 2018. Facultad de Humanidades y Ciencias de la Educación. Universidad Nacional de La Plata
}

\author{
Lucia Dibene \\ Universidad Nacional de La Plata, Argentina \\ luciabelend@gmail.com \\ Luciana Falco \\ Universidad Nacional de La Plata, Argentina \\ luciaana.f@gmail.com
}

El día 24 de Noviembre del 2018 tuvo lugar, en la Facultad de Humanidades y Ciencias de la Educación de la Universidad Nacional de La Plata, la jornada Enseñar matemáticas en aulas que incluyen alumnos con y sin discapacidad. Dicho encuentro se enmarca dentro de las acciones desplegadas desde el Proyecto Promocional de Investigación y Desarrollo (PPID) denominado: Aportes de la Didáctica de la Matemática para el estudio de la inclusión de personas con discapacidad en escuelas comunes (H025, 2017-2018) ${ }^{1}$. El mismo tiene como propósito contribuir al campo en construcción de la educación inclusiva desde los aportes de la Didáctica de la Matemática, con el objetivo de efectivizar el derecho a la educación de los alumnos con discapacidad en aulas comunes.

La Jornada fue organizada por la Secretaria de Posgrado, el Departamento de Ciencias Exactas y Naturales, el Departamento de Ciencias de la Educación y la Cátedra Didáctica de la Matemática de la Facultad de Humanidades y Ciencias de la Educación. La coordinación estuvo a cargo de Claudia Broitman, Pilar Cobeñas, Mónica Escobar y Verónica Grimaldi. Entre sus propósitos centrales podemos mencionar la necesidad de: socializar y difundir el trabajo realizado desde el proyecto, visibilizar algunas de las problemáticas existentes en torno a la enseñanza de la matemática a alumnos con discapacidad, problematizar la importancia de construir una educación inclusiva y generar un espacio de diálogo, discusión e intercambio entre docentes de educación especial, docentes de educación común y educadores de matemática.

Durante el transcurso de la jornada se realizaron diferentes ponencias a cargo de los integrantes del equipo de investigación y de docentes e investigadores invitados. A continuación presentamos los aspectos más salientes de las mismas.

Pilar Cobeñas, Juan Cobeñas y Clara Goitia ${ }^{2}$ iniciaron la jornada historizando la lucha por los derechos de las personas con discapacidad y presentando las bases de la Educación Inclusiva. Los disertantes hicieron particular mención a la Declaración de Salamanca (ONU, 1994), a partir de la cual la discapacidad es entendida bajo el modelo social; y a la Convención Internacional sobre los Derechos de las Personas con Discapacidad (2006) impulsada por las Naciones Unidas, firmada por la Argentina en 2008. El artículo 24 de este último se refiere específicamente a la educación inclusiva.

Los Estados Partes reconocen el derecho de las personas con discapacidad a la educación. Con miras a hacer efectivo este derecho sin discriminación y sobre la base de la igualdad de oportunidades, los Estados Partes asegurarán un sistema de educación inclusivo a todos los niveles así como la enseñanza a lo largo de la vida. (CDPCD, art. 24). ${ }^{3}$

Clara Goitia enfatizó que, a diferencia de la perspectiva del modelo médico, el modelo social entiende que las barreras que obstaculizan o impiden la inclusión plena de las personas con discapacidad son producidas por la sociedad y no son atribuibles a la discapacidad en sí misma. Como parte central de la ponencia, Juan 
Cobeñas y Clara Goitia compartieron sus experiencias escolares en el sistema educativo, cuestión que es retomada por Verónica Grimaldi y enriquecida desde su perspectiva docente al acompañar la trayectoria educativa de Juan Cobeñas.

En su presentación, Verónica Grimaldi compartió su experiencia como profesora de matemática en un aula inclusiva, destacando que la misma se constituyó en una herramienta para problematizar las prácticas escolares. Proyectó un video que presenta una escena de un aula de escuela secundaria en la que un alumno con discapacidad se encuentra resolviendo una actividad de matemática. ${ }^{4}$ Se observa claramente que lo acompaña un intérprete y que hace uso del sistema de comunicación aumentativa alternativa como un apoyo necesario para interactuar con sus compañeros y profesores. Frente a una duda, se produce un intercambio con la profesora, quien interviene brindando las aclaraciones necesarias sin reemplazar al estudiante en la resolución de la tarea. La disertante enfatizó que seleccionó esta escena para destacar que no tiene nada de "extraordinario". Se trata de un estudiante interactuando con su profesora como todos sus compañeros. Encuentra en esta escena familiar un buen ejemplo de lo que entiende por educación inclusiva.

A continuación, Claudia Broitman presentó las ideas centrales del enfoque de enseñanza de las matemáticas en las que se apoya el proyecto Aportes de la Didáctica de la Matemática para el estudio de la inclusión de personas con discapacidad en escuelas comunes. De manera general, menciona que se inscribe en el campo de la didáctica de la matemática de tradición francesa surgido en la década del ochenta a partir de los trabajos de Guy Brousseau (1994, 2007), Yves Chevallard (1997) y Gerard Vergnaud (1990), entre otros. Desde esta perspectiva, lejos de pensar la enseñanza de la matemática como una transmisión de mecanismos a ser aprendidos de memoria, se considera que no se trata de enseñarles a los niños los rudimentos de unas definiciones, de algunos teoremas, de algunas técnicas, sino de formarlos como sujetos más ampliamente. Los alumnos deben pensar por sí mismos y comportarse como sujetos matemáticos, como sujetos de la cultura, como individuos autónomos intelectualmente. Asimismo, desde este enfoque se asume el rol fundamental de las interacciones entre pares durante los procesos constructivos de conocimiento matemático.

Seguidamente, Mónica Escobar presentó las principales líneas de investigación que se desarrollan en forma simultánea al interior del proyecto. La primera busca analizar las formas de enseñanza y las concepciones didácticas vigentes en aulas de educación especial; la segunda, propone indagar acerca de las experiencias, posibilidades y obstáculos que los docentes identifican en la planificación y desarrollo de las clases de matemáticas en aulas de escuelas comunes cuya matrícula está integrada por alumnos con y sin discapacidad; finalmente, la última línea de investigación apunta a relevar prácticas de enseñanza en aulas plurigrado de escuelas rurales a las que asisten alumnos con y sin discapacidad. Destacó que, dada la amplitud y complejidad de la problemática estudiada, fue necesario incluir en el equipo la mirada y los saberes de distintos campos profesionales: profesores de Matemática, profesores en Ciencias de la Educación, acompañantes terapéuticos, psicólogos, especialistas en Didáctica de la Matemática, especialistas en Educación Inclusiva, docentes de educación común y de educación especial. Las tres ponencias que se describen a continuación, presentaron los propósitos de cada una de las líneas de investigación mencionadas, las acciones desarrolladas, los principales hallazgos y los nuevos interrogantes.

En primer lugar, expusieron Claudia Broitman, Emilio González, Lucía Dibene y Luciana Falco en representación del equipo que estudia la enseñanza de la matemática en escuelas de educación especial. Al igual que el resto de los equipos, la información fue relevada a partir de indagación bibliográfica, observaciones de clases y entrevistas a docentes, en este caso, de escuelas especiales urbanas. Se presentaron resultados y reflexiones alrededor de algunos fenómenos observados en torno al sentido asignado a la matemática, las actividades, el papel de lo lúdico y el contenido matemático.

En segundo lugar, presentaron su trabajo los integrantes del equipo que estudia la enseñanza de la matemática en aulas de escuelas comunes urbanas a las que asisten alumnos con y sin discapacidad: Pilar Cobeñas, Verónica Grimaldi, Agustina Villanueva, Guadalupe Herrero, Laura Murua y Mariana Filardi. Luego de hacer referencia de manera general al marco jurídico de la educación inclusiva, describieron las 
observaciones de clase realizadas. La presentación tomó como ejes las formas de trabajo áulico, las relaciones entre adultos presentes en el aula, las estrategias de enseñanza y las actividades matemáticas propuestas. En su presentación incluyeron extractos de registros de clase y ejemplos de las situaciones propuestas a los estudiantes.

Por último, expuso el equipo que estudia la enseñanza de la matemática en aulas plurigrado de escuelas rurales a las que asisten alumnos con y sin discapacidad. Mónica Escobar, Itatí Blanco, Lourdes Salas y Lourdes Tardío presentaron el análisis de algunos de los datos relevados, enmarcando las observaciones y testimonios de los entrevistados en las particularidades y desafíos que presenta la enseñanza de la matemática cuando se produce en aulas plurigrado. Sin dejar de reconocer las barreras para la inclusión que se presentan en estos contextos, destacaron las potencialidades que identifican en estas aulas y que podrían favorecer la instalación de prácticas inclusivas.

Finalizada la presentación del proyecto y los avances de cada subequipo, se expusieron los tres trabajos que completaron la jornada.

Mónica Escobar y Verónica Grimaldi hicieron referencia a la educación inclusiva en el Nivel Inicial. Este nivel, señalan, representa el primer contacto que los niños tienen con una institución educativa y el inicio de su trayectoria escolar, sin embargo, también puede dar origen a trayectorias de exclusión. En este sentido, analizaron ciertas prácticas que pueden favorecer u obstaculizar la inclusión de todos los niños y sus posibilidades de avanzar en la escolaridad. Para ello apelan al marco legal del nivel, deteniéndose particularmente en el artículo 14 de la Resolución $174 / 12^{5}$ que explicita: "la trayectoria escolar de niños y niñas en el nivel inicial no podrá ser alterada bajo la idea de permanencia o repitencia”. A continuación compartieron fragmentos de clases de matemática en dos jardines de infantes urbanos. ${ }^{6}$ En ambas salas, la matrícula estaba integrada por alumnos con y sin discapacidad. Destacaron que en estas clases todos los alumnos se encontraban resolviendo problemas ligados al mismo contenido, cuestión que favoreció diversas interacciones en los pequeños grupos y en los espacios de trabajo colectivo. A su vez, describieron y analizaron los diversos apoyos que ofrecieron las docentes, maestras integradoras y acompañantes terapéuticos para optimizar el trabajo matemático de los alumnos.

Por su parte, Paula Trillini y Andrea Novembre compartieron y analizaron el trabajo que vienen desarrollando en relación a las clases de matemática en aulas de nivel medio a las que asisten alumnos con y sin discapacidad. Se centraron en los nuevos desafíos profesionales que enfrentaron al asumir la enseñanza desde una perspectiva inclusiva. En su relato resaltaron la importancia del trabajo en equipo a nivel institucional y estableciendo vínculos con la familia y los distintos actores que acompañan la trayectoria educativa de los estudiantes. Asimismo, destacaron la importancia de la construcción de conocimiento matemático por parte del alumno y en colaboración con otros. También hicieron referencia a los apoyos que pusieron a disposición del alumno, particularmente subrayaron el uso de las TIC en esta práctica.

Por último, disertó un investigador invitado, Pablo Correa, quien presentó su proyecto de tesis referido al aprendizaje de la geometría en alumnos ciegos. ${ }^{7}$ El mismo se constituye con el fin de contribuir al campo de la didáctica de la matemática y al campo de la educación inclusiva de manera conjunta.

Finalizadas las ponencias, se habilitó un espacio de intercambio en el que los asistentes manifestaron sus inquietudes y reflexiones sobre la temática abordada a lo largo de la jornada. La alta concurrencia y el interés sostenido hasta la finalización del encuentro dan cuenta de que este era un espacio necesario. Así lo reconocieron los responsables de la organización, quienes resaltaron a su vez la importancia de generar espacios de intercambio y divulgación académica y la relevancia de estos estudios para avanzar hacia la garantía plena y efectiva del derecho a la educación de las personas con discapacidad. 


\section{Bibliografía}

Brousseau, G. (1994). “Los diferentes roles del maestro”. En Parra, C., y Saiz, I. (comp.). Didáctica de las Matemáticas. Aportes y Reflexiones. Buenos Aires: Paidós.

Brousseau, G. (2007). Introducción a la Teoría de las Situaciones Didácticas. Buenos Aires: Libros del Zorzal.

Chevallard, Y. (1997): La Transposición Didáctica. Buenos Aires: Aique.

Organización de Naciones Unidas (1994). Declaración de Salamanca.

Organización de Naciones Unidas (2006). Convención sobre los Derechos de las Personas con Discapacidad y Protocolo Facultativo.

Vergnaud, G. (1990). La théorie des champs conceptuels. En: Recherches en Didactique des Mathématiques, 10 (2 y 3 ), 133-170. (Traducción mimeografiada).

\section{Notas}

1. El proyecto de investigación está dirigido por la Dra. Claudia Broitman e integrado por Itatí Blanco, Marianela Boriosi, Johanna Dávila, Lucía Dibene, Mónica Escobar, Luciana Falco, Mariana Filardi, Emilio González, Marisol Goñi, Verónica Grimaldi, Guadalupe Herrero, Ana Paula Lemos, María Luján Miranda, Laura Murúa, Lourdes Salas, Inés Sancha, Lourdes Tardío y Agustina Villanueva.

2. Los tres expositores son miembros de la Asociación Azul. Puede accederse al sitio oficial a través de: http://www.asoci acionazul.org.ar/

3. Con el propósito de resguardar el cumplimiento de tales transformaciones, en la Argentina se ha conformado el Grupo Artículo 24 para la educación inclusiva. Puede accederse al sitio oficial a través de: http://www.grupoart24.org/

4. El material se titula "Interiores (mi Cuerpo y Yo)". El fragmento proyectado inicia en el minuto 5:22 y finaliza en el minuto 6:12. Se encuentra disponible en: http://encuentro.gob.ar/programas/4983

5. La Resolución $N^{\circ} 174$, Pautas federales para el mejoramiento de la enseñanza y el aprendizaje y las trayectorias escolares en el nivel inicial, primario y modalidades y su regulación, fue aprobada en 2012 por el Consejo Federal de Educación (Argentina). Disponible en: http://www.me.gov.ar/consejo/resoluciones/res12/174-12.pdf

6. Los fragmentos de video mencionados forman parte de los micrositios sobre la enseñanza del sistema de numeración (htt $\mathrm{p}: / /$ servicios2.abc.gov.ar/lainstitucion/sistemaeducativo/educacioninicial/sistema_numeracion/) y sobre la enseñanza de la geometría (http://servicios2.abc.gov.ar/lainstitucion/sistemaeducativo/educacioninicial/geometriaeneljardin/) elaborados por la Dirección Provincial de Educación Inicial de la provincia de Buenos Aires.

7. El proyecto de tesis se enmarca en la Maestría en Ciencias Exactas y Naturales de la Facultad de Humanidades y Ciencias de la Educación de la Universidad Nacional de La Plata. El proyecto está dirigido por la Dra. Claudia Broitman y codirigido por la Dra. Pilar Cobeñas. 